\title{
Structured Reporting of Solid and Cystic Pancreatic Lesions in CT and MRI: Consensus-Based Structured Report Templates of the German Society of Radiology (DRG)
}

\author{
Strukturierte Befundung von soliden und zystischen \\ Pankreasläsionen im CT und MRT: Konsensus-basierte \\ Befundvorlagen der Deutschen Röntgengesellschaft (DRG)
}

Authors

Thorsten Persigehl1', Matthias Baumhauer², Bettina Baeßler1, 27, Lukas Philipp Beyer ${ }^{3}$, Marc Bludau4, Christiane Bruns ${ }^{4}$, Alexander Christian Bunck ${ }^{1}$, Christoph-Thomas Germer ${ }^{5}$, Lars Grenacher ${ }^{6}$, Thomas Hackländer ${ }^{7}$, Stefan Haneder ${ }^{1}$, Stefan Heinrich ${ }^{8}$, Philipp Heusch ${ }^{9}$, Matthias Hoffmann ${ }^{10}$, Markus S. Juchems ${ }^{11}$, Jan Robert Kröger ${ }^{1}$, Elmar Kotter ${ }^{12}$, Günter Layer ${ }^{13}$, David Maintz ${ }^{1}$, Jan Menke ${ }^{14}$, Moritz Palmowski ${ }^{15,}{ }^{28}$, Dimitrios Pantelis ${ }^{16}$, Daniel Pinto dos Santos ${ }^{1}$, Felix Christoph Popp ${ }^{4}$, Nico Schäfer ${ }^{17}$, Heinz-Peter Schlemmer ${ }^{18}$, Andreas G. Schreyer ${ }^{19}$, Florian Siedek ${ }^{1}$, Dirk Stippel ${ }^{20}$, Martin Völker ${ }^{21}$, Dirk Thomas Waldschmidt ${ }^{22}$, Tim Frederik Weber ${ }^{23}$, Moritz von Winterfeld ${ }^{24}$, Christian Wybranski ${ }^{1}$, Thomas Zander ${ }^{25}$, Johannes Wessling ${ }^{26}$

Affiliations

1 Department of Radiology, University of Cologne, Germany

2 Mint Medical GmbH, Heidelberg, Germany

3 Department of Radiology, University Medical Center Regensburg, Germany

4 General, Visceral and Cancer Surgery, University of Cologne, Germany

5 Department of General, Visceral, Vascular and Pediatric Surgery, Würzburg University Hospital, Würzburg, Germany

6 Imaging and Prevention Center, Conradia Radiology Munich, Germany

7 Department of Radiology, HELIOS-Universitätsklinikum Wuppertal, Germany

8 General, Visceral and Transplantation Surgery, Mainz University, Mainz, Germany

9 Department of Radiology, University Hospital Düsseldorf, Germany

10 Department of General and Visceral Surgery, Raphaelsklinik Münster, Germany

11 Department of Radiology, Hospital Konstanz, Germany

12 Department of Radiology, Freiburg University Hospital, Freiburg, Germany

13 Department of Radiology, Hospital Ludwigshafen, Germany

14 Department of Diagnostic Radiology, University Hospital Göttingen, Germany

15 Department of Radiology, Radiology Baden-Baden, Baden-Baden, Germany

16 Department of Surgery, Sankt-Marien-Hospital Bonn GmbH, Bonn, Germany

17 Department of Surgery, Hospital Leverkusen, Germany

18 Department of Radiology, Deutsches

Krebsforschungszentrum, Heidelberg, Germany
19 Institute for Diagnostic and Interventional Radiology, Brandenburg Medical School Theodor Fontane, Brandenburg a. d. Havel, Germany

20 Department of General, Visceral and Cancer Surgery, University of Cologne, Germany

21 DRG, German Roentgen Society “Deutsche Röntgengesellschaft”, Berlin, Germany

22 Department of Gastroenterology and Hepatology, University of Cologne, Germany

23 Department of Radiology, University Hospital Heidelberg, Germany

24 Institute of Pathology, University Hospital Heidelberg, Germany

25 Department of Internal Medicine, University of Cologne, Germany

26 Department of Radiology, Clemens-Hospital GmbH Münster, Germany

27 Institute of Diagnostic and Interventional Radiology, University Hospital Zurich, Switzerland

28 Institute of Experimental Molecular Imaging (ExMI), RTW Aachen, Germany

Key words

pancreas, structured reporting, template, MRI, CT

received 29.08.2019

accepted 25.01.2020

Bibliography

DOI https://doi.org/10.1055/a-1150-8217

Published online: 2020

Fortschr Röntgenstr 2020; 192: 641-655

(C) Georg Thieme Verlag KG, Stuttgart · New York

ISSN 1438-9029 
Correspondence

Dr. Thorsten Persigehl

Radiology, University Hospital Cologne, Kerpener Str. 62,

50937 Köln, Germany

Tel.: ++49/2 21/47896045

thorsten.persigehl@uk-koeln.de

\section{ABSTRACT}

Background Radiological reports of pancreatic lesions are currently widely formulated as free texts. However, for optimal characterization, staging and operation planning, a wide range of information is required but is sometimes not captured comprehensively. Structured reporting offers the potential for improvement in terms of completeness, reproducibility and clarity of interdisciplinary communication.

Method Interdisciplinary consensus finding of structured report templates for solid and cystic pancreatic tumors in computed tomography (CT) and magnetic resonance imaging (MRI) with representatives of the German Society of Radiology (DRG), German Society for General and Visceral Surgery (DGAV), working group Oncological Imaging (ABO) of the German Cancer Society (DKG) and other radiologists, oncologists and surgeons.

Results Among experts in the field of pancreatic imaging, oncology and pancreatic surgery, as well as in a public online survey, structured report templates were developed by consensus. These templates are available on the DRG homepage under www.befundung.drg.de and will be regularly revised to the current state of scientific knowledge by the participating specialist societies and responsible working groups.

Conclusion This article presents structured report templates for solid and cystic pancreatic tumors to improve clinical staging (cTNM, ycTNM) in everyday radiology.

Key Points:

- Structured report templates offer the potential of optimized radiological reporting with regard to completeness, reproducibility and differential diagnosis.

- This article presents consensus-based, structured reports for solid and cystic pancreatic lesions in CT and MRI.
- These structured reports are available open source on the homepage of the German Society of Radiology (DRG) under www.befundung.drg.de.

\section{Citation Format}

- Persigehl T, Baumhauer M, Baeßler B et al. Structured Reporting of Solid and Cystic Pancreatic Lesions in CT and MRI: Consensus-Based Structured Report Templates of the German Society of Radiology (DRG). Fortschr Röntgenstr 2020; 192: 641-655

\section{ZUSAMMENFASSUNG}

Hintergrund In der klinischen Radiologie werden aktuell radiologische Befundberichte bei Pankreasläsionen weitverbreitet als Freitexte formuliert. Für die optimale klinische Versorgung, das Staging und die Operationsplanung sind mannigfaltige Informationen erforderlich, die mitunter nicht umfänglich erfasst werden. Die strukturierte Befundung bietet hier das Potenzial einer Verbesserung in Bezug auf Vollständigkeit, Reproduzierbarkeit sowie Klarheit der interdisziplinären Kommunikation.

Methode Interdisziplinäre Erstellung von strukturierten Befundvorlagen für solide und zystische Pankreastumoren in der Computertomografie (CT) und in der Magnetresonanztomografie (MRT) mit Vertretern der Deutschen Röntgengesellschaft (DRG), der Deutschen Gesellschaft für Allgemein- und Viszeralchirurgie (DGAV), der Arbeitsgemeinschaft Onkologische Bildgebung (ABO) der Deutschen Krebsgesellschaft (DKG) sowie weiteren Radiologen, Onkologen und Chirurgen. Ergebnisse Unter Experten auf dem Gebiet der Pankreasbildgebung, onkologischen Versorgung und Pankreaschirurgie sowie in einer öffentlichen Online-Umfrage wurden strukturierte Befundvorlagen im Konsensus-Verfahren entwickelt, die im weiteren Verlauf regelmäßig durch die beteiligten Fachgesellschaften und verantwortlichen Arbeitsgemeinschaften auf den aktuellen Stand der wissenschaftlichen Erkenntnis angepasst werden sollen.

Zusammenfassung Dieser Artikel stellt strukturierte Befundvorlagen für solide und zystische Pankreastumoren vor, die im radiologischen Alltag helfen sollen, das klinische Staging (cTNM, ycTNM) zu verbessern.

\section{Background}

Oncology reports in clinical radiology are currently largely written as free text [1]. Consequently, the structure, level of detail, terminology, and clarity of these reports vary depending on the experience and expertise of the reporting physician. It can be difficult for the referring physician to obtain complete and reproducible information from the radiology report. Therefore, it can be unclear whether certain findings are non-existent or were simply not included in the report. Important information for decisions, for example, between a curative versus a palliative treatment approach may not be sufficiently provided so that another time-intensive review of the image data is necessary.
Structured reporting can improve the completeness, reproducibility, and clarity of interdisciplinary communication [1, 2]. Moreover, structured report templates can ensure consistent and higher report quality. This is true particularly when the correct detection and description of a pathology as well as the systematic and complete application of report criteria are essential for an optimal treatment decision for an individual patient. Reports of pancreatic tumors fulfill these requirements and necessities from the perspective of oncologists and surgeons in a special way. Brook et al. showed that both the intelligibility of the report as well as the completeness of the report with respect the provision of sufficient information for surgical planning and the assessment of resectability can profit significantly from structured reporting 
(free text vs. structured report independently rated by surgeons with $31 \% / 43 \% / 25 \%$ vs. $96 \% / 69 \% / 98 \%$ ) [3]. The results of a study by Kabadi et al. highlight the importance of the use of standardized terminology. Differences in the perception and interpretation of free-text reports could be significantly reduced by using structured report templates with defined selection options with standardized terminology [4].

Only a limited number of structured oncology report templates are currently available. In 2008, the Radiological Society of North America (RSNA) began compiling a collection of report templates in text and HTML/MRRT format that are available online at www.radreport.org. In 2014, a structured report template for ductal adenocarcinoma of the pancreas was published by the American Society of Abdominal Radiology (SAR) and the American Pancreatic Association (ASA) in consensus. It was adopted by the National Comprehensive Cancer Network (NCCN) and the American Joint Committee on Cancer (AJCC). In Germany, there is currently no national interdisciplinary consensus-based template for the staging of pancreatic tumors on computed tomography $(\mathrm{CT})$ and magnetic resonance imaging (MRI).

This article presents for the first time a structured and interdisciplinary German report template for solid and cystic pancreatic tumors developed in consensus with a public survey. The goal is to improve the clarity and significance of the radiology report with regard to staging (CTNM, ycTNM), treatment decisions and possible surgical planning.

\section{Creation of structured report templates by the German Society of Radiology (DRG)}

The German Society of Radiology (DRG) addressed the lack of quality-assured report templates in German and began assembling a collection of German report templates. These templates are available via the homepage www.befundung.drg.de for free non-commercial use (Creative Commons License). This license allows the download and distribution of the template with the DRG named as the creator. In concrete terms, interested users can download DRG templates and use them locally or a webbased tool provided free of charge by the working group for information technology (AGIT) (EasyRad, IFTM GmbH, Solingen) can be used for editing and the generated report can be copied to the own report or RIS/HIS.

In a consensus meeting a first version of structured report templates for solid pancreatic lesions on CT and MRI were created in consensus with 19 DRG representatives from the working group for oncological imaging, the working group for gastrointestinal and abdominal imaging, the working group for information technology (AGIT), the German Society for General and Visceral Surgery (DGAV), the working group for oncological imaging of the German Cancer Society (DKG), the Center for Integrated Oncology (CIO) Cologne/Bonn, the German Cancer Research Center (DKFZ) and additional radiologists, oncologists, and surgeons (including participants M. B. and M. V. as neutral observers). The current literature, guidelines, and requirements of the NCCN and AJCC were taken into consideration [5-9]. In a subsequent public consensus survey, the members of the DRG and DGAV were invi- ted to participate in the Delphi method online via www.survey monkey.de/r/pankreas and to vote on 56 questions and enter comments. An additional 93 radiologists and visceral surgeons participated resulting in a total participation of over 100 people (consensus results in the online supplement). The lead authors (T.P. and J.W.) compiled the consensus results, the online voting results, and all comments in a manuscript and added a description of cystic pancreatic lesions. This manuscript was discussed again by the experts and published as an initial version of a consensus paper with the presented structured report templates for solid and cystic pancreatic lesions.

The templates will be regularly reviewed by the relevant professional societies and responsible working groups and adjusted to the current state of scientific knowledge. In addition, a possibility to provide feedback was created on the DRG homepage under www.befundung.drg.de and supported by the AGIT at the email address: agit-sr@googlegroups.com.

\section{General structure of the structured report templates}

The structured report templates presented here are divided into a description and evaluation section. The description section includes questions regarding possible prior imaging, image quality, histology/lgG4 followed by information regarding tumor (T-stage) with possible local organ infiltration and vascular changes, lymph node status ( $\mathrm{N}$-stage), and additional abdominal structures (M-stage). To optimize the communication of findings, retrieval, and transparency, the serial number and image number or alternatively the table position should be specified for the relevant findings. To reduce the number of different templates, special features of CT and MRI were not used in the characterization of the finding (for example, instead of hypodense for CT and hypointense for MRI, simplified to hypo-).

The most probable radiological diagnosis should be specified in the assessment possibly followed by differential diagnoses (consensus was not reached among experts or in the online survey regarding specification of the subjective reliability of the finding based on the Likert score of 1-5). In the case of suspicion of a malignant process, the clinical cTNM stage based on the relevant imaging should be additionally specified (high consensus among experts and online). Alternatively, the prefixes ctTNM and mriTNM can be used.

The structured report templates intentionally do not contain imaging recommendations, imaging algorithms, or protocols for performing examinations. Refer to the current national guidelines and international guidelines (AWMF, UEG, ESMO, ACR, SAR and NCCN) [6-11].

\section{Structured report templates}

Templates for solid and cystic pancreatic tumors on CT and MRI are provided in the following ( $\bullet$ Fig. 1,2 ). Multiple selection options are provided in some cases. In individual cases, if the options are not applicable, it is possible to enter the finding as free text. In 
sections marked with the symbol "+", multiple options can be selected or can be duplicated in the case of multiple lesions. In the case of metastases, the explicit naming and measurement of a maximum of 2 lesions per organ based on RECIST 1.1 seems sufficient. Further analogous lesions can be combined into one group with brief specification of the anatomical segment.

\section{Basic clinical knowledge regarding pancreatic lesions in pathology}

In general, pancreatic lesions can be classified as solid, cystic, and mixed solid-cystic. Among solid tumors, ductal adenocarcinoma is the most common pancreatic tumor ( $90 \%$ ). Solid acinar cell carcinomas, neuroendocrine tumors of the pancreas (NET), and solid pseudopapillary neoplasms are significantly rarer. Among cystic lesions, reactive, i.e., post-inflammatory pseudocysts, must be differentiated from neoplastic lesions. The latter include intraductal papillary mucinous neoplasms (IPMNs) as well as the rarer mucinous-cystic neoplasms (MCNs) and serous-cystic neoplasms (SCNs).

\section{Classification of solid pancreatic tumors}

In the case of solid pancreatic tumors, tumors of the exocrine pancreas can be differentiated from those of the endocrine pancreas. The most common solid pancreatic tumor is ductal adenocarcinoma which comprises approx. $90 \%$ of tumors of the exocrine pancreas and sometimes occurs as a mixed type ductalacinar cell carcinoma. Acinar cell carcinoma and solid-pseudopapillary neoplasm (SPN; syn. Frantz tumor), which can exhibit secondary pseudocystic degeneration, and neuroendocrine tumors of the pancreas (NETs), which can rarely also appear purely cystic, are much less common. Endocrine pancreatic tumors include a heterogeneous group of tumor entities with varying hormone activity and variable malignancy potential. A differentiation is made between hormonally active and hormonally inactive pancreatic tumors, with inactive NETs being much more common and tending to be malignant. Hormonally active tumors usually have specific clinical symptoms with insulinomas (hypoglycemia) and gastrinomas (Zollinger-Ellison syndrome) being most common. VIPomas (Vernor-Morrison syndrome), glucagonomas, somatostatinomas, and ACTHomas (extraadrenal Cushing's syndrome) are less common. Pancreatic NETs can occur as part of multiple endocrine neoplasia syndrome (MEN 1), as gastrinomas in approx. $75 \%$ of cases and as insulinomas in approx. $25 \%$ of cases. Gastrinomas are usually larger $(>5 \mathrm{~cm})$ and insulinomas are usually smaller $(<2 \mathrm{~cm})$ when diagnosed. Hypervascularized solid pancreatic tumors, particularly in the tail of the pancreas, must be differentiated from intra-/parapancreatic accessory spleens as a variant, while hypervascularized solid pancreatic tumors in the entire pancreas must be differentiated from distant metastases, particularly in the case of a longer relapse-free period after clearcell renal cell carcinoma (10 years or longer). Moreover, acute pancreatitis as well as a rare extranodal lymphoma manifestation should be considered in the case of focal or diffuse enlargement of the pancreas and pronounced lymphadenopathy.

\section{Classification of cystic pancreatic tumors}

Cystic pancreatic tumors include a broad group of different lesions. According to their origin, histology, and malignancy potential, these can be classified as I) epithelialized and non-epithelialized pancreatic lesions, II) neoplastic and non-neoplastic pancreatic tumors and III) benign and (pre-)malignant pancreatic lesions [12-15]. > Fig. 3, > Table 1 show the most common cystic pancreatic tumors $[12,14]$.

Non-epithelialized, non-neoplastic pseudocysts and retention cysts after acute pancreatitis or as part of chronic pancreatitis are most common (approx. $40 \%$ of cases). The most common epithelialized and neoplastic cystic pancreatic tumor is the intraductal papillary mucinous neoplasm (IPMN) (approx. $25 \%$ of cases) followed by the mucinous-cystic neoplasm (MCN) and the serouscystic neoplasm (usually serous cystandenoma, extremely rarely serous cystandenocarcinoma). Serous cystadenomas can increase in size over their course. In the case of mucinous-cystic neoplasm, MCN with minimal or intermediate dysplasia is differentiated from MCN with severe dysplasia and MCN with associated invasive carcinoma. Solid pseudopapillary neoplasms (SPNs) with pseudocystic degeneration and a low malignancy potential are less common $(<5 \%)$. Intraductal papillary mucinous neoplasms (IPMNs) can be classified analogous to MCN based on the degree of dysplasia or an associated carcinoma. IPMN is further classified as main duct IPMN, side-branch duct IPMN, or mixed type IPMN. Branch duct IPMN represents the most common IPMN subtype which is increasingly recognized with the increase in imaging of the abdomen. In principle, every IPMN corresponds to a malignant precursor lesion with main duct IPMN having a higher risk for malignant transformation to an invasive ductal adenocarcinoma (see below).

Moreover, there are a number of additional primary cystic, neoplastic and non-neoplastic pancreatic lesions and central necrotic/ cystic and mixed solid-cystic variants of neoplastic pancreatic tumors with cystic degeneration, e.g. cystic ductal adenocarcinomas, cystic neuroendocrine neoplasms (NETs), and rare cystic hamartomas and cystic teratomas [12-14] ( $\triangleright$ Table 2 ). In rare cases, distant metastases in the pancreas can also appear cystic or mixed solid-cystic.

With respect to macropathology, cystic pancreatic lesions can be described as microcystic, macrocystic, oligocystic, unilocular, or cystic with solid portions [6, 12-14]:

- Microcystic lesions (microcyst on microcyst) are characteristic for serous cystic neoplasms (SCNs), sometimes unilocular and oligocystic serous cysts. When diagnosed, serous cystadenomas (SCNs) are usually $2-16 \mathrm{~cm}$ and the individual microcysts are 1-20 mm, typically with a honeycomb pattern. In SCN, small intralesional cysts can only be differentiated on MRI, while wall calcifications and the central scar with possible calcification can be better detected on CT. SCNs can increase in size over time.

- Macrocystic lesions ("macrocyst with intralesional cysts and/or septations”) include mucinous cystic neoplasms (MCNs, cystadenomas or cystadenocarcinomas), intraductal papillary mucinous neoplasms (IPMNs), and pseudocysts after pancreatitis. At the time of diagnosis, mucinous cystic neoplasms (MCNs) are usually $3-23 \mathrm{~cm}$ and the individual macrocysts are $>10 \mathrm{~mm}$. MCNs and pseudocysts usually have a thicker wall compared to IPMNs. 


\section{Solid pancreatic lesion on CT/MRI}

Description:

+ No relevant prior images

+ Prior images from:

General information:

Image quality: good/average/poor

Histology/lgG4: still pending/confirmed

Solid pancreatic lesion:

Pancreatic parenchyma: normal/edamatous/lipotropic/signs of chronic pancreatitis

Number of lesions: singular/approx.__multifocal

+ Location: Head of the pancreas/uncinate process/body of the pancreas/tail of the pancreas

Size: $\quad \mathrm{mm}$ (series/image number)

Characterization: solid/mixed solid and cystic/necrotic

Contrast enhancement compared to pancreatic tissue:

Arterial: hypo-/iso-/hyper-

Venous: hypo-/iso-/hyper-

Pancreatic duct: unremarkable/prestenotic dilatation with max. diameter:

Common bile duct: unremarkable/prestenotic dilatation with max. diameter: /stent normal

Index lesion maximum size: _ $\mathrm{mm}$

(Exocrine information: T1: $\leq 2 \mathrm{~cm}$ (T1a: $\leq 0.5 \mathrm{~cm} / T 1 b<1 \mathrm{~cm} / T 1 \mathrm{c}: \leq 2 \mathrm{~cm}) / T 2: \leq 4 \mathrm{~cm} / T 3:>4 \mathrm{~cm} / T 4$ : Vascular infiltration)

(Neuroendocrine information: T1: $<2 \mathrm{~cm} / T 2: 2-4 \mathrm{~cm} / T 3$ : $>4 \mathrm{~cm} / T 4$ : Organ or vascular infiltration)

Index lesion with infiltration of adjacent organs: stomach/spleen/duodenum/jejunum/colon/kidney/left/right adrenal gland

Index lesion with vascular infiltration: no/yes (from celiac trunk, superior mesenteric artery and/or common hepatic artery, see below for details)

Vascular involvement of the primary tumor (specified in degrees of the circumference)

Aorta (ventral): $\mathrm{no} / \leq 180^{\circ} />180^{\circ} /$ deformation

Celiac trunk: no $\leq 180^{\circ} />180^{\circ}$ /deformation

Common hepatic artery: no/ $\leq 180^{\circ} />180^{\circ}$ /deformation

Proper hepatic artery: no/ $\leq 180^{\circ} />180^{\circ}$ /deformation

Gastroduodenal artery (close to the origin): no/ $\leq 180^{\circ} />180^{\circ} /$ deformation

Superior mesenteric artery: no/ $\leq 180^{\circ} />180^{\circ} /$ deformation

Splenic artery: no/ $\leq 180^{\circ} />180^{\circ} /$ deformation

Anatomical vascular variants: no/accessory right hepatic artery/common hepatic artery from the superio mesenteric artery/other: _Infiltration: no/ $\leq 180^{\circ} />180^{\circ} /$ deformation

Splenic vein: no/ $\leq 180^{\circ} />180^{\circ}$ /deformation/thrombosis

Superior mesenteric vein: no/ $\leq 180 \%$ / $180 \%$ deformation/first jejunal branch infiltrated/thrombosis

Portal vein: $\mathrm{no} / \leq 180^{\circ} />180^{\circ} /$ deformation/thrombosis/free text, e.g. cavernous transformation

Atherosclerosis of the celiac trunk: no/stenosis approx. \%

Atherosclerosis of the superior mesenteric artery: no/stenosis approx. \%

Locoregional lymph node: no suspicious lymph nodes/suspicious lymph nodes number:_, max. _mm.

Location: (series/image number)

Distant lymph nodes: no suspicious lymph nodes/suspicious lymph nodes number: max. mm. Location:

Ascites: no/yes: minimal/pronounced/peritoneal implants Location:

+ Liver: unremarkable/detectable:

+ Liver metastases: Total number: in segment:

+ Measurement of max. 2 lesions according to RECIST 1.1:

L01:_ (series/image number)

L02: (series/image number)

+ Cyst in the segment:

+ Hemangioma in the segment:

+ Other liver lesions: Free text

Bile ducts/gallbladder: unremarkable/cholestasis/choledocholithiasis/cholecystolithiasis

Spleen: unremarkable/craniocaudal splenomegaly max. $\mathrm{cm}$.

+ Kidney/ureter: unremarkable/detectable:

+ Renal cyst Bosniak: _/free text

Adrenal gland: unremarkable/free text

Colon: unremarkable/free text

Pelvic organs: unremarkable/free text

Bone: unremarkable/free text

Recorded basal lung segments: unremarkable/free text

Assessment:

Pancreatic cancer in the

+ Suspected pancreatic cancer in the

+ cTxNxMx (lymphogenic/hepatic/osseous/pulmonary)

- Fig. 1 Structured report template for solid pancreatic lesions on CT and MRI. 


\section{Cystic pancreatic lesion on CT/MRI:}

\section{Description:}

+ No relevant prior images

+ Prior images from:

General information:

Image quality: good/average/poor

Histology: still pending/confirmed

Cystic pancreatic lesion:

Number of lesions: singular/approx.__multifocal

+ Location: head of the pancreas/uncinate process/body of the pancreas/tail of the pancreas

Characterization: cystic/microcystic/macrocystic/oligocystic/unilocular/cystic with solid segments

Size:__ mm (series/image number)

Contour: sharp/unsharp

Wall: none/thin/thic

Septations: no/yes

Solid components (intramural nodules): no/yes: central/peripheral

Contrast enhancement: no/yes

Calcifications: no/yes

Duct association: none/main duct/branch duct/combined main duct and branch duct

Pancreatic duct: unremarkable/prestenotic dilatation with max. diameter:

Common bile duct: unremarkable/prestenotic dilatation with max. diameter: _stent normal

Index lesion maximum size: _ $\mathrm{mm}$

(Exocrine information: $\mathrm{T1}: \leq 2 \mathrm{~cm}(\mathrm{~T} 1 \mathrm{a}: \leq 0.5 \mathrm{~cm} / \mathrm{T} 1 \mathrm{~b}<1 \mathrm{~cm} / \mathrm{T} 1 \mathrm{c}: \leq 2 \mathrm{~cm}) / T 2: \leq 4 \mathrm{~cm} / \mathrm{T} 3:>4 \mathrm{~cm} / \mathrm{T} 4$ : Vascular infiltration)

(Neuroendocrine information: $11:<2 \mathrm{~cm} / \mathrm{T} 2: 2-4 \mathrm{~cm} / \mathrm{T3}$ : $>4 \mathrm{~cm} / \mathrm{T} 4$ : Organ or vascular infiltration)

Index lesion with infiltration of adjacent organs: stomach/spleen/duodenum/jejunum/colon/kidney/left/right adrenal gland

Index lesion with vascular infiltration: no/yes (from celiac trunk, superior mesenteric artery and/or common hepatic artery, see below for details)

Vascular involvement of the primary tumor (specified in degrees of the circumference)

Aorta (ventral): no/ $\leq 180^{\circ} />180^{\circ} /$ deformation

Celiac trunk: no/ $\leq 180^{\circ} />180^{\circ} /$ deformation

Common hepatic artery: no/ $\leq 180^{\circ} />180^{\circ} /$ deformation

Proper hepatic artery: no/ $\leq 180^{\circ} />180^{\circ} /$ deformation

Gastroduodenal artery (close to the origin): no/ $\leq 180^{\circ} />180^{\circ}$ /deformation

Superior mesenteric artery: no/ $\leq 180^{\circ} />180^{\circ} /$ deformation

Splenic artery: no/ $\leq 180^{\circ} />180^{\circ} /$ deformation

Anatomical vascular variants: no/accessory right hepatic artery/common hepatic artery from the superior mesenteric artery/other:__ Infiltration: no/ $\leq 180^{\circ} />180^{\circ} /$ deformation

Splenic vein: $\mathrm{no} / \leq 180^{\circ} />180^{\circ} /$ deformation/thrombosis

Superior mesenteric vein: no/ $\leq 180^{\circ} />180^{\circ} /$ deformation/first Jejunal branch infiltrated/thrombosis

Portal vein: no/ $\leq 180^{\circ} />180^{\circ}$ /deformation/thrombosis/free text, e.g. cavernous transformation

Atherosclerosis of the celiac trunk: no/stenosis approx. \%

Atherosclerosis of the superior mesenteric artery: no/stenosis approx. \%

Locoregional lymph node: no suspicious lymph nodes/suspicious lymph nodes. Number: max. mm. Location:__ (series/image number)

Distant lymph nodes: no suspicious lymph nodes/suspicious lymph nodes. Number:_, max._mm. Location:

Ascites: no/yes: minimal/pronounced/peritoneal implants. Location:

+ Liver: unremarkable/detectable:

+ Liver metastases: Total number: _ in segment:

+ Measurement of max. 2 lesions according to RECIST 1.1

L01: (series/image number)

L02: (series/image number)

+ Cyst in the segment:

+ Hemangioma in the segment:

+ Other liver lesions: Free text

Bile ducts/gallbladder: unremarkable/cholestasis/choledocholithiasis/cholecystolithiasis

Spleen: unremarkable/craniocaudal splenomegaly max. $\mathrm{cm}$.

+ Kidney/ureter: unremarkable/detectable:

+ Renal cyst Bosniak: __free text

Adrenal gland: unremarkable/free text

Colon: unremarkable/free text

Pelvic organs: unremarkable/free text

Bone: unremarkable/free text

Recorded basal lung segments: unremarkable/free text

Assessment:

+ Known [Etiology] (e.g. serous cystadenoma/mucinous cystadenoma/IPMN type: branch duct/main duct/ mixed) in the

+ Suspected [Etiology] in the

+ cTxNxMx (hepatic/pulmonary/osseous/lymphogenic)

- Fig. 2 Structured report template for cystic pancreatic lesions on CT and MRI. 


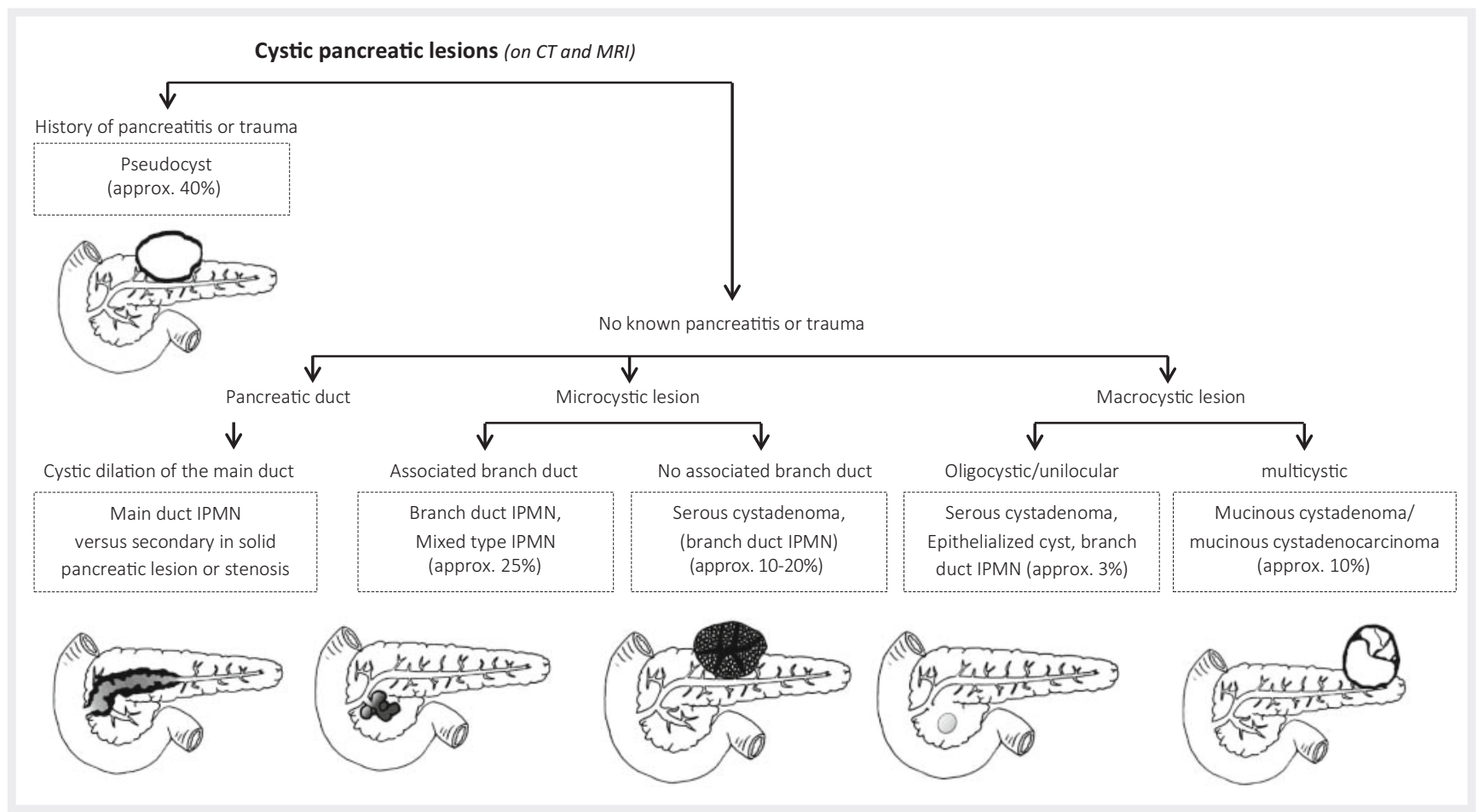

- Fig. 3 The most frequent cystic pancreatic lesions on CT and MRI (modified from [12]).

- Table 1 The most frequent cystic pancreatic lesions and their malignancy potential (modified from [14]).

\begin{tabular}{|l|l|l|}
\begin{tabular}{l|l|} 
cystic pancreatic \\
lesion
\end{tabular} & $\begin{array}{l}\text { frequency } \\
\text { in } \%\end{array}$ & malignancy potential \\
\hline Pseudocyst & 40 & benign \\
\hline IPMN & 25 & $\begin{array}{l}\text { variable, according to position and } \\
\text { risk factors: }\end{array}$ \\
\cline { 1 - 1 } branch duct & & low \\
\hline IPMN & & high \\
\hline main duct IPMN & & intermediate \\
\hline mixed type IPMN & & intermediate \\
\hline MCN & 10 & extremely low \\
\hline SCN & 10 & low \\
\hline SPN & $<5$ & \\
\hline
\end{tabular}

- Oligocystic lesions (“cyst with minimal septations”) are a special type of serous cystic neoplasm (SCN) and IPMN (sometimes undetectable branch duct) as well as pseudocysts. - Unilocular cysts ("simple singular cyst”) have no septae and no solid portion and often correspond to a pseudocyst as well as a retention cyst, monocystic SCN, and more rarely a branch duct IPMN or types of rare epithelialized, non-neoplastic pancreatic cysts, e. g. congenital cysts and lymphoepithelial cysts. Multiple epithelialized cysts often occur in Hippel-Lindau syndrome, autosomal-dominant polycystic kidney disease (ADPKD), and cystic fibrosis/mucoviscidosis and are usually $<15 \mathrm{~mm}$.
- Table 2 Overview of cystic pancreatic lesions (modified from [14]).

\section{Non-neoplastic pancreatic cysts}

epithelialized:

- congenital cyst

- retention cyst

- enterogenous cyst

- duodenal wall cyst

- endometrial cyst

not epithelialized:

- pseudocyst

- parasitic cyst
- lymphoepithelial cyst

\section{Neoplastic pancreatic cysts}

epithelialized:

- IPMN

- MCN

- SCN

- SPN

- acinar cell cystadenoma

- cystic teratoma

- cystic hamartoma

- ductal adenocarcinoma, rarely purely cystic

- acinar cell carcinoma, rarely purely cystic

- neuroendocrine tumor, rarely purely cystic not epithelialized:

- lymphangioma

- sarcoma, cystic

- hemangioma

- metastases, cystic
- Cystic lesions with a solid portion (“mixed cystic-solid lesions”) can be a consequence of primary cystic pancreatic tumors (such as MCN and IPMN), cystic solid tumors (as in NETs), necrotic solid tumors (like ductal adenocarcinoma, acinar cell tumors or metastases), primary cystic-solid tumors (e. g. SPN) or pseudocysts after pancreatitis. 
When determining differential diagnoses, cystic pancreatic lesions can also be further categorized on the basis of age of predilection (SPN commonly seen in young women; MCN tends to occur in pre-/perimenopausal women; SCN commonly seen in older women, IPMN more commonly seen in older men and women), sex (MCN almost exclusively seen in women), location (MCN primarily seen in the caudal region, SCN primarily seen in the head/corpus), number (IPMN, congenital cysts, and pseudocysts tend to be multifocal), morphology (SCN tends to be microcystic while MCN and pseudocysts tend to be macrocystic) or on the basis of communication with the pancreatic duct (branch duct IPMN with associated side-branch duct) and history (incidental finding in pre-/perimenopausal women possible MCN versus pseudocyst after pancreatitis). However, deviations from this always need to be taken into consideration.

\section{Basic clinical knowledge of anatomy}

Pancreatic lesions are categorized based on their location as lesions of the head of the pancreas (caput pancreatis, to the right of the left edge of the superior mesenteric vein), of the body of the pancreas (corpus pancreatis, between the left edge of the superior mesenteric vein and the left edge of the aorta) and of the tail of the pancreas (cauda pancreatis, starting from the left edge of the aorta) ( $\triangleright$ Fig. 4 [16]). In particular cases, for a more precise description, the uncinate process can be differentiated as part of the head of the pancreas and the neck of the pancreas, ventral to the superior mesenteric vein and the portal vein.

\section{Pancreatic duct and variants}

The pancreatic duct (ductus pancreaticus) normally measures up to $3 \mathrm{~mm}$ and usually opens together with the common bile duct at the major duodenal papilla (papilla of Vater) into the duodenum. An additional drainage duct can be present as a variant (syn. accessory pancreatic duct) that opens cranial into the duodenum at the minor duodenal papilla (papilla of Santorini).

In $5-10 \%$ of cases, pancreas divisum can be present as a variant with the main pancreatic duct opening into the minor duodenal papilla via the duct of Santorini and a small branch duct from the head of the pancreas/uncinate process opening separately into the major duodenal papilla via the duct of wirsungianus, socalled complete pancreas divisum. In the case of an incomplete pancreas divisum, there is a smaller connecting duct. Pancreas divisum is a risk factor for acute or chronic pancreatitis.

In annular pancreas a circle of pancreatic tissue surrounds the duodenum and is associated with an increased risk for duodenal stenosis. Moreover, partial/complete agenesis and ectopic pancreatic tissue can be detected in rare cases but must not be confused with a pancreatic tumor.

\section{Vascular supply}

The celiac trunk, common hepatic artery, gastroduodenal artery, and superior pancreaticoduodenal artery and an arcade to the inferior pancreaticoduodenal artery via the superior mesenteric

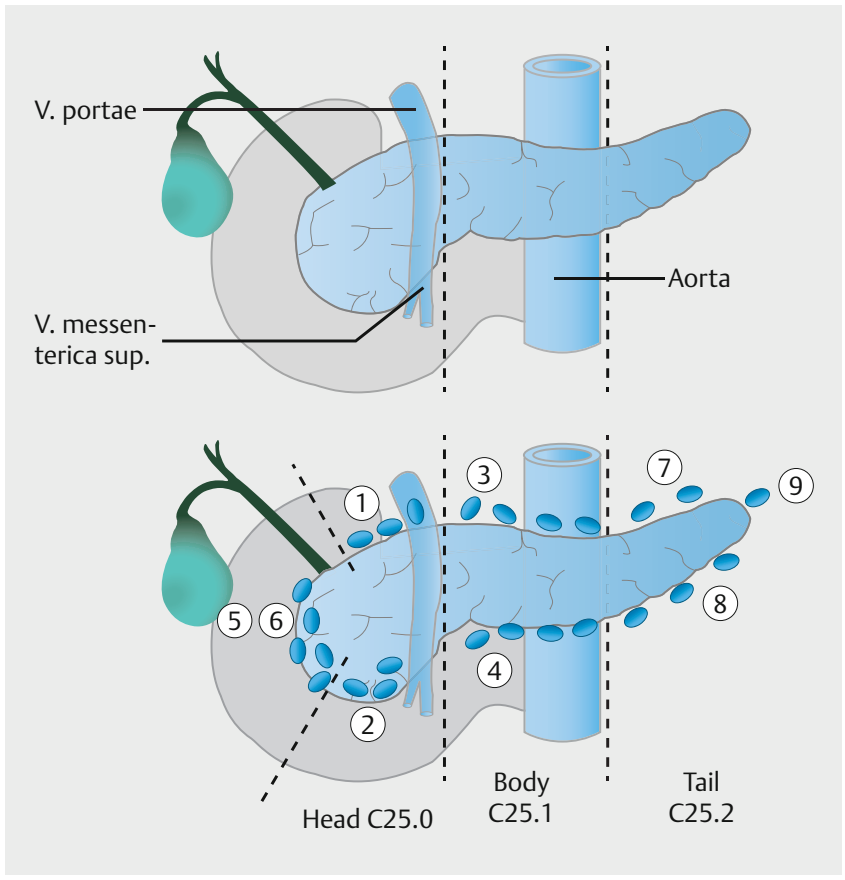

- Fig. 4 Anatomy of the pancreas with the locoregional lymph node stations (from [16]). 1: Superior pancreaticoduodenal lymph nodes, 2: Inferior pancreaticoduodenal lymph nodes, 3: Celiac lymph nodes, 4: Superior mesenteric lymph nodes, 5: Cystic lymph nodes, 6: Hepatic lymph nodes, 7: Superior pancreatic lymph nodes, 8: Inferior pancreatic lymph nodes, 9: Splenic lymph nodes.

artery supply the head of the pancreas. The body of the pancreas and tail of the pancreas are supplied by the splenic artery and its branches, the rami pancreatici. The head of the pancreas drains via the pancreaticoduodenal veins into the superior mesenteric vein and portal vein. The body and tail of the pancreas drain via the pancreatic veins into the splenic vein.

\section{Lymphatic drainage}

The lymph of the head of the pancreas preferably drains into the locoregional lymph nodes, the superior and inferior pancreaticoduodenal lymph nodes, and from there into the celiac lymph nodes and the superior mesenteric lymph nodes ( $\triangleright$ Fig. 4 ). The body and tail of the pancreas drain into the locoregional lymph nodes, the superior and inferior pancreatic lymph nodes, and then into the celiac lymph nodes and the superior mesenteric lymph nodes, and in the region of the tail of the pancreas into the splenic lymph nodes.

\section{Basic clinical knowledge of TNM classifications}

The structured report templates presented here are based on the TNM classification according to the $8^{\text {th }}$ edition of the American Joint Committee on Cancer (AJCC) for exocrine pancreatic cancer and neuroendocrine tumors (NETs) of the pancreas that are classified by a separate TNM system. 


\section{Exocrine pancreatic cancer}

\section{TNM classification of exocrine pancreatic cancer (AJCC, $8^{\text {th }}$ edition)}

\begin{tabular}{|c|l|}
\hline TX & No statement about primary tumor possible \\
\hline T0 & No evidence of a primary tumor \\
\hline T1 & Tumor $\leq 2 \mathrm{~cm}$ in greatest dimension \\
\hline T1a & Tumor $\leq 0.5 \mathrm{~cm}$ in greatest dimension \\
\hline T1b & Tumor $>0.5 \mathrm{~cm}$ and $\leq 1 \mathrm{~cm}$ in greatest dimension \\
\hline T1c & Tumor $>1$ and $\leq 2 \mathrm{~cm}$ in greatest dimension \\
\hline T2 & Tumor $>2 \mathrm{~cm}$ and $\leq 4$ cm in greatest dimension \\
\hline T3 & Tumor $>4 \mathrm{~cm}$ in greatest dimension \\
\hline T4 & Tumor infiltrating celiac trunk, superior mesenteric artery \\
\hline and $/ o r$ common hepatic artery \\
\hline N0 & No reliable statement about lymph node metastases possible \\
\hline N1 & No regional lymph node metastases \\
\hline N2 & Metastases in 1 to 3 regional lymph nodes \\
\hline MX & Metastases in 4 or more regional lymph nodes \\
\hline M0 & No distant metastases \\
\hline M1 & Distant metastases \\
\hline
\end{tabular}

\section{Stages of exocrine pancreatic cancer}

\begin{tabular}{|l|l|l|l|}
\hline Stage 0 & Tis & N0 & M0 \\
\hline Stage IA & $\mathrm{T} 1$ & N0 & M0 \\
\hline Stage IB & $\mathrm{T} 2$ & $\mathrm{~N} 0$ & $\mathrm{M} 0$ \\
\hline Stage IIA & $\mathrm{T} 3$ & $\mathrm{~N} 0$ & $\mathrm{M} 0$ \\
\hline Stage IIB & $\mathrm{T} 1, \mathrm{~T} 2, \mathrm{~T} 3$ & $\mathrm{~N} 1$ & $\mathrm{M} 0$ \\
\hline Stage III & $\mathrm{T} 1, \mathrm{~T} 2, \mathrm{~T} 3$ & $\mathrm{~N} 2$ & $\mathrm{M}$ \\
\hline & $\mathrm{T} 4$ & Every N & $\mathrm{M} 0$ \\
\hline Stage IV & Every T & Every N & $\mathrm{M} 1$ \\
\hline
\end{tabular}

$\mathrm{N}$-stage: The regional lymph nodes for tumors of the head of the pancreas include lymph nodes along the common bile duct, the common hepatic artery, the portal vein, the pylorus of the stomach, the posterior and anterior pancreaticoduodenal arcade, and the lymph nodes along the superior mesenteric vein and along the right lateral wall of the superior mesenteric artery ( $\triangleright$ Fig.4). In tumors of the body and tail, the regional lymph nodes are located along the common hepatic artery, around the celiac trunk and along the splenic artery to the splenic hilum.

M-stage: Distant metastases are often in non-locoregional lymph nodes, the liver, and the peritoneum and more rarely in the lung, pleura, bone, brain, and soft tissues (the latter is not rare in an advanced tumor stage).
Neuroendocrine tumors (NETs) of the pancreas:

\section{TNM classification of neuroendocrine tumors of the pancreas (AJCC, $8^{\text {th }}$ Edition)}

\begin{tabular}{|c|l|}
\hline TX & No reliable statement about primary tumor possible \\
\hline T0 & No evidence of a primary tumor \\
\hline T1 & Tumor $<2 \mathrm{~cm}$ in greatest dimension \\
\hline T2 & Tumor $2-4 \mathrm{~cm}$ in greatest dimension \\
\hline T3 & $\begin{array}{l}\text { Tumor }>\text { } 4 \mathrm{~cm} \text { in greatest dimension or tumor infiltrating the } \\
\text { duodenum or the common bile duct }\end{array}$ \\
\hline T4 & $\begin{array}{l}\text { Tumor infiltrating adjacent organs (stomach, spleen, colon, } \\
\text { adrenal gland) or the vascular wall of the great vessels } \\
\text { (celiac trunk or superior mesenteric artery) }\end{array}$ \\
\hline NX & No reliable statement about lymph node metastases possible \\
\hline N0 & No regional lymph node metastases \\
\hline N1 & Regional lymph node metastases \\
\hline MX & No reliable statement regarding distant metastases possible \\
\hline M0 & No distant metastases \\
\hline M1 & Distant metastases \\
\hline & M1a: Liver metastases \\
\hline & M1b: Distant metastases to a non-hepatic organ (e. g. lung) \\
\hline M1c: Liver and non-hepatic organs \\
\hline
\end{tabular}

\section{Stages of neuroendocrine tumors of the pancreas}

\begin{tabular}{|l|l|l|l|}
\hline Stage I & T1 & N0 & M0 \\
\hline Stage II & T2, T3 & N0 & M0 \\
\hline Stage III & T4 & N0 & M0 \\
\hline & Every T & N1 & M0 \\
\hline Stage IV & Every T & Every N & M1 \\
\hline
\end{tabular}

$\mathrm{N}$-stage: The regional lymph nodes for neuroendocrine tumors of the head of the pancreas and the body and tail of the pancreas correspond to the classification and location in ductal adenocarcinoma.

M-stage: Distant metastases are frequently seen in the liver and more rarely in the lung and bone.

\section{Basic clinical knowledge regarding pancreatic lesions in radiology}

When analyzing CT and MRI images, the pancreas must be systematically evaluated, detected pancreatic lesions must be characterized, and clinically relevant changes must be documented in detail in a structured manner when possible.

1. General evaluation of the pancreatic parenchyma

2. Detection of a pancreatic lesion or possible early signs 
3. Anatomical location of the lesion, relationship to the pancreatic duct and measurement

4. Morphological characterization (solid, cystic, mixed solid-cystic)

5. Information regarding surrounding structures for the assessment of surgical resectability (arterial and venous situation, common bile duct, and pancreatic duct, lymph nodes, adjacent organs, distant metastases)

6. Radiological differential diagnosis

\section{Evaluation of the pancreatic parenchyma}

The morphology of the parenchyma provides information about the presence of acute or chronic pancreatitis, pancreatic lipomatosis, and global versus segmental atrophy. In case of acute pancreatitis, edamatous swelling of the pancreas with a normal-sized pancreatic duct is typically seen in early stage, in later stages diffuse interstitial/exudative edema into the adjacent fat tissue or later on necrosis of the pancreatic parenchyma and adjacent fat tissue could be present. Global atrophy often with small calcifications and global dilatation of the pancreatic duct and occasionally segmental atrophy is seen in chronic pancreatitis. Focal biliary pancreatitis of the head of the pancreas in choledocholithiasis as well as groove pancreatitis in the space between the head of the pancreas and the duodenum sometimes cannot be reliably differentiated from cancer of the head of the pancreas. However, the acute clinical symptoms and detection of a prepapillary concretion with simultaneous thickening of the duodenal wall and a main finding on the right side of the head of the pancreas can indicate an acute or chronic inflammatory origin. However, this is complicated by the fact that acute pancreatitis can mask pancreatic cancer, chronic pancreatitis is a risk factor for pancreatic cancer and reliable differentiation on imaging is sometimes not possible. In contrast, diffuse organ swelling with elimination of the lobe structure and the adjacent "halo", elevated IgG4 levels and other associated autoimmune diseases, such as sclerosing cholangitis or biliary liver cirrhosis, are typically seen in autoimmune pancreatitis.

\section{Detection of the pancreatic lesion or possible early signs}

Solid pancreatic tumors appear isodense or isointense with respect to the pancreatic parenchyma in the various contrast agent phases in approx. 10-15\% of cases and are thus sometimes not able to be detected, particularly when $<2 \mathrm{~cm}$. Secondary imaging signs must be observed here:

- Structural abnormality with a homogeneous lesion within the lobe structure or focal elimination of the typical lobed organ contour possibly with bulging.

- Focal edema into the adjacent fat tissue as a possible early sign of local tumor infiltration. Focal pancreatitis must always be considered as a differential diagnosis here.

- Abrupt dilatation of the pancreatic duct without a definitive cause with upstream dilatation and possible atrophy of the adjacent pancreatic parenchyma.

- Dilatation of the pancreatic duct and the common bile duct starting in the head of the pancreas (double duct sign). However, this is a nonspecific sign with numerous differential diag- noses including prepapillary choledocholithiasis, sphincter of Oddi dysfunction, papillary stenosis due to scarring, or papillary carcinoma.

\section{Anatomical location, relationship to the pancreatic duct and measurement}

The anatomical location and the possible relationship to the pancreatic duct are particularly important when determining differential diagnoses, for example, there is a high probability of branch duct IPMN in the case of a cystic lesion with an associated sidebranch duct. Moreover, the exact location with the direct position in relation to surrounding structures is important for the surgeon in the case of possible resection. In radiology, lesions are preferably measured in the axial (transverse) slice orientation. Cystic lesions like IPMNs can sometimes be better measured and visualized in their entirety in coronal slice orientation.

\section{Morphological characterization}

Pancreatic lesions are primarily classified as solid, cystic, or mixed solid-cystic. Classic ductal adenocarcinoma usually has a hyalinized stroma which delimits the hypodense/hypointense tumor from the normal parenchyma particularly in the late arterial phase, the so-called pancreatic parenchyma phase. Depending on the degree of differentiation of adenocarcinomas, the contrast behavior can adjust to normal parenchyma contrast enhancement behavior so that well-differentiated tumors in particular can be missed despite being of a substantial size.

In contrast, neuroendocrine tumors are hypervascularized in the majority of cases compared to the pancreatic parenchyma. Intrapancreatic accessory spleens should be considered as a differential diagnosis in the case of circumscribed, highly vascularized lesions located in the outermost portion of the tail of the pancreas. Metastases should also be considered as a differential diagnosis in the case of multifocality and pancreatic metastases in the case of clear cell renal cell carcinoma and malignant melanoma.

Accordingly, cystic pancreatic tumors demonstrate typical cystic imaging findings on CT and MRI. MRI sometimes helps to determine the primary cystic character of the lesion in T2-weighted sequences. This is particularly true for microcystic lesions with septations that are often misinterpreted as solid tumors on CT due to their hypodense appearance. Solid tumor segments, such as mural nodules as a risk factor for malignancy in IPMN, can be detected based on the contrast agent dynamics, and cystic or necrotic degeneration of solid pancreatic tumors can be visualized as mixed solid-cystic.

\section{Information regarding surrounding structures and possible resectability}

Exact image analysis of the surrounding structures (arterial and venous vessels, common bile duct and pancreatic duct, lymph nodes, neighboring organs, distant metastases, and relevant additional findings) are essential for the evaluation of resectability and possible surgical planning. In the early stage, perivascular, lymphatic and perineural spreading is seen in pancreatic cancer. Therefore, special attention must be paid to possible vascular contact and infiltration into the retroperitoneal fat tissue in the dorsal 

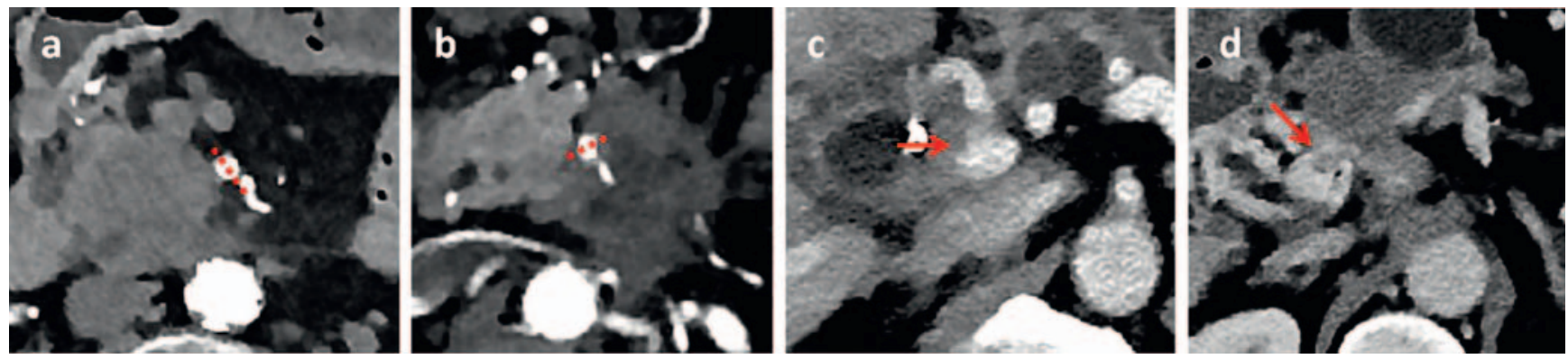

Fig. 5 Possible vascular involvement with A) Abutment/contact $\leq 180^{\circ}$ to the AMS, B) Encasement/contact $>180^{\circ}$ of the AMS, C) Deformity with compression of the portal vein and D) Infiltration with tumor thrombus in the portal vein when the VMS is occluded. AMS = superior mesenteric artery, VMS = superior mesenteric vein.

direction to the celiac trunk, the AMS or the aorta in the primary diagnosis and in postoperative follow-up [17]. To evaluate resectability, vascular involvement is decisive for the T-stage and is categorized as follows ( $\bullet$ Fig. 5) [5]:

1. Abutment $/$ contact $\leq 180^{\circ}=$ contact corresponding to vascular contact $\leq 180$ degrees and low risk of vascular infiltration and possible $\mathrm{R} 0$ resectability.

2. Encasement $/$ contact $>180^{\circ}=$ encasement corresponding to vascular contact $>180$ degrees; with an increased risk of vascular infiltration, $\mathrm{R} 0$ resectability seems difficult here.

3. Deformity = irregularity corresponding to a very high risk of vascular infiltration with possible infiltration into the lumen or at least deformation of the lumen.

4. Thrombus=tumor thrombus, corresponding to vascular invasion with intraluminal tumor tissue, to be differentiated from a "bland" thrombus, for example in the case of stenosis.

Encasement, deformity and tumor thrombus are image criteria for a high or very high risk of vascular infiltration. Edema into the peripancreatic and thus also perivascular fat tissue can also occur as a result of desmoplastic reactions, secondary pancreatitis in tumorous obstruction of the pancreatic duct or after ERCP and can simulate vascular infiltration. For this reason radiological staging should ideally be performed prior to planned ERCP or stent implantation. After neoadjuvant radiotherapy/chemotherapy, posttherapeutic changes to perivascular fat tissue ("fat stranding") make radiological differentiation of avital fibrosis from vital tumor infiltration extremely difficult or impossible.

When describing a local finding, organ infiltration should be included even if it is sometimes not relevant for the T-stage but is important for surgical planning with respect to possible resection of the stomach, spleen, duodenum, jejunum, colon, kidneys, or adrenal glands to be performed.

Radiological evaluation of lymph node metastases (N-stage) is difficult. A short axis diameter (SAD) of $\geq 10 \mathrm{~mm}$ is typically used here as a morphological imaging criterion. In addition, an irregular contour, an inhomogeneous texture and/or central necrosis as well as an increased number of smaller lymph nodes bordering a pancreatic carcinoma are at least suspicious for lymphogenic me- tastasis $(\mathrm{N}+)$. In simplified terms, lymph node metastases located in the surgical region are to be considered locoregional metastases $(\mathrm{N}+)$ and those outside of this region are to be considered distant metastases $(\mathrm{M}+)$.

In addition to local infiltration, the exclusion of distant metastases (M-stage) is also important for assessing operability, with limited metastases (oligometastasis) increasingly not necessarily being a contraindication for surgery, particularly in the case of regression or stable condition after neoadjuvant radiotherapy/chemotherapy. Preferred metastasis sites are distant lymph nodes, liver, peritoneum, and lung.

For individual surgical planning, tumorous changes as well as the vascular anatomy with possible variants (corresponding to the Michels classification) must be documented ( $\mathbf{F i g . 6}$ ). This is true particularly for variations in the origin of the right hepatic artery and common hepatic artery which can originate from the superior mesenteric artery (AMS) as a variant. Moreover, stenoses of the celiac trunk and the AMS should be described and quantified. Unidentified high-grade stenosis at the origin of the celiac trunk or the AMS with retrograde flow in the gastroduodenal artery can result in liver necrosis, intestinal ischemia, or impaired wound healing with insufficiency, for example in routine ligature of the gastroduodenal artery during a Whipple procedure.

\section{Radiological differential diagnosis}

In the differential diagnosis, morphological characterization (solid, cystic, or mixed solid-cystic) as well as close interdisciplinary collaboration and consideration of clinical, anatomical, and methodological information are particularly important and help to narrow down the differential diagnosis, particularly:

1. Patient age and sex

2. History of prior diseases, comorbidities, or tumor markers

3. Results of ERCP, EUS, FNA or brush cytology

- Fig. 7 shows an overview of the most common solid, mixed solid-cystic and cystic pancreatic lesions under consideration of additional clinical factors and deviations from the typical appearance. 


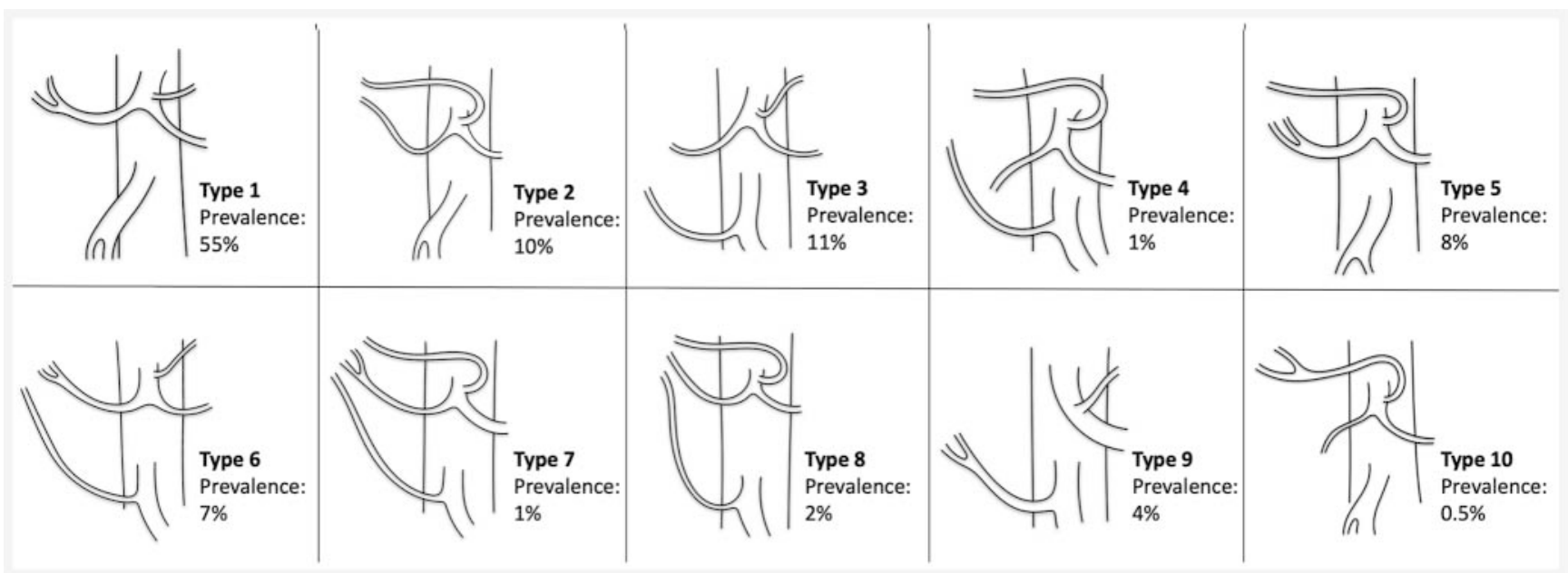

- Fig. 6 Variants of the celiac trunk and superior mesenteric artery (AMS). Michels classification with type 1: Common hepatic artery until the gastroduodenal artery, then proper hepatic artery with division into the right and left hepatic arteries. Type 2: Left hepatic artery via left gastric artery. Type 3: Right hepatic artery from the AMS. Type 4: simultaneously types 2 and 3. Type 5: accessory left hepatic artery additionally from the left gastric artery. Type 6: accessory right hepatic artery additionally from the AMS. Type 7: simultaneously types 5 and 6. Type 8: Right hepatic artery from the AMS and accessory left hepatic artery. Type 9: Common hepatic artery from the AMS. Type 10: Common hepatic artery from the left gastric artery.

\section{Basic clinical knowledge of surgical resectability}

The guidelines of the National Comprehensive Cancer Network (NCCN) are accepted worldwide for the assessment of the resectability of pancreatic tumors ( Table 3 ) $[8,11,17]$. These are used both for ductal adenocarcinomas and for other tumor entities like neuroendocrine tumors (NETs) and cystic neoplasms of the pancreas. According to the NCCN guidelines, pancreatic cancers are divided into three categories:

- Resectable

- Borderline resectable

- Not resectable

Resectable pancreatic tumors are locally limited tumors with a reasonable chance of R0 resection. Borderline resectable refers to pancreatic tumors infiltrating neighboring structures in a circumscribed manner with a relatively high probability of an R1 resection but with a possible survival advantage. Non-resectable pancreatic tumors are tumors with distant metastases or locally advanced tumors without distant metastases for which there is a high probability that surgery with $\mathrm{R} 1 / \mathrm{R} 2$ resection will not result in a survival advantage due to the local infiltration pattern.

According to the NCCN guidelines, a detailed radiology report on the arterial and venous vascular situation, organ infiltration, and distant metastases is necessary for better assessment of the resectability of pancreatic cancers by visceral surgeons. Attention should also be paid to the extent of venous infiltration. Therefore, tumor infiltration of the first jejunal branch into the superior mesenteric vein (VMS) can prevent possible resection or require individual vascular reconstruction via anastomosis or interposition grafts by an experienced surgeon. In the case of infiltration, the patient can be viewed by the visceral surgeon primarily as "nonresectable" but with the option of secondary reevaluation after radiotherapy/chemotherapy. The complexity of the NCCN guidelines is taken into advantage in the structured report templates presented here with checklists for all relevant arteries and veins. A structured radiology report should provide the visceral surgeon with the best possible description of all findings. Assessment of resectability and surgical planning are the responsibility of an experienced visceral surgeon and sometimes require joint image interpretation and demonstration in an interdisciplinary tumor board.

Intraductal papillary mucinous neoplasms (IPMNs) represent a special situation in relation to the indication for surgery. There are various recommendations in this regard most recently in the European Guidelines of the European Study Group on Cystic Tumors of the Pancreas [6] ( $\triangleright$ Table 4). Also in the case of neuroendocrine and cystic masses of the pancreas, the same technical requirements apply in principle but the indications for resection vary depending on the presumed pathology, e. g. mucinous versus serous cystadenoma.

\section{Basic clinical knowledge of surgical techniques}

The surgical technique used in pancreatic surgery is largely based on the location and presumed pathology of the pancreatic tumor. In the case of tumors in the head of the pancreas, uncinate process, neck of the pancreas, or right-sided proximal body of the pancreas, partial pancreaticoduodenectomy using the Whipple procedure or Traverso-Longmire technique with pylorus preservation is typically performed. If located in the left-sided distal body of the pancreas or tail of the pancreas, left-sided pancreatic resection can be performed. In special constellations such as in localized neuroendocrine tumors (NETs), intraductal papillary mucinous neoplasms (IPMNs), solid pseudopapillary neoplasms (SPN; so-called Frantz tumor) or mucinous cystadenomas (MCNs), cen- 




- Fig.7 Radiological algorithm for diagnosis of the most common solid, mixed cystic-solid and cystic pancreatic lesions on CT and MRI. 
- Table 3 Resectability criteria according to the National Comprehensive Cancer Network (NCCN) (Version 2/2018, Update 1/2019 [11]).

\begin{tabular}{|c|c|c|}
\hline resectability & arterial & venous \\
\hline resectable & $\begin{array}{l}\text { no tumor contact with celiac trunk }[\mathrm{TC}] \text {, superior mesenteric } \\
\text { artery }[\mathrm{AMS}] \text {, or common hepatic artery }[\mathrm{AHC}]\end{array}$ & $\begin{array}{l}\text { no tumor contact with the superior mesenteric vein [VMS] or } \\
\text { portal vein }[\mathrm{PA}] \text { or } \leq 180^{\circ} \text { contact without contour irregularity } \\
\text { of the vein }\end{array}$ \\
\hline $\begin{array}{l}\text { "borderline" } \\
\text { resectable }\end{array}$ & $\begin{array}{l}\text { tumor in the head of the pancreas or uncinate process: } \\
\text { - solid tumor contact with the common hepatic artery } \\
\text { without extension to the celiac trunk or the bifurcation of } \\
\text { the hepatic artery with possible safe and complete resec- } \\
\text { tion and reconstruction } \\
\text { " solid tumor contact with the superior mesenteric artery } \\
\text { with } \leq 180^{\circ} \\
\text { - solid tumor contact with artery variant (e. g. accessory } \\
\text { right hepatic artery, variant origin of the left hepatic } \\
\text { artery, variant origin of the common hepatic artery. The } \\
\text { type of variant and extent of tumor contact should be } \\
\text { specified since they can affect surgical planning. } \\
\text { tumor in the body and tail of the pancreas: } \\
\text { - solid tumor contact with the celiac trunk with } \leq 180^{\circ} \\
\text { - solid tumor contact with the celiac trunk with }>180^{\circ} \\
\text { without involvement of the aorta or the intact gastroduo- } \\
\text { denal artery so that a modified operation is possible if } \\
\text { needed. }\end{array}$ & $\begin{array}{l}\text { solid tumor contact with the superior mesenteric vein or } \\
\text { portal vein with }>180^{\circ} \text {, contact with } \leq 180^{\circ} \text { with contour } \\
\text { irregularity of the vein or thrombosis of the vein with } \\
\text { preserved vein proximal and distal to the affected vascular } \\
\text { segment so that safe and complete resection and recon- } \\
\text { struction is possible. } \\
\text { - solid tumor contact with the inferior vena cava [VCI]. }\end{array}$ \\
\hline $\begin{array}{l}\text { not } \\
\text { resectable }\end{array}$ & $\begin{array}{l}\text { - distant metastases (including distant lymph node } \\
\text { metastases) } \\
\text { tumor in the head of the pancreas or uncinate process: } \\
\text { " solid tumor contact with the superior mesenteric artery } \\
>180^{\circ} \\
\text { - solid tumor contact with the celiac trunk }>180^{\circ} \\
\text { tumor in the body and tail of the pancreas: } \\
\text { - solid tumor contact with }>180^{\circ} \text { with the mesenteric artery } \\
\text { or celiac trunk } \\
\text { " solid tumor contact with the celiac trunk and involvement } \\
\text { of the aorta }\end{array}$ & $\begin{array}{l}\text { tumor in the head of the pancreas or uncinate process: } \\
\text { " non-reconstructable superior mesenteric vein or portal vein } \\
\text { in the case of tumor involvement or occlusion (as a result of } \\
\text { tumor thrombus or bland thrombus) } \\
\text { " tumor contact with the most proximal jejunal branch } \\
\text { draining into the portal vein } \\
\text { tumor in the body and tail of the pancreas: } \\
\text { " non-reconstructable superior mesenteric vein or portal vein } \\
\text { in the case of tumor involvement or occlusion (as a result of } \\
\text { tumor thrombus or bland thrombus) }\end{array}$ \\
\hline
\end{tabular}

tral pancreatic resection or tumor enucleation with or without preservation of the spleen can be performed. In the case of multifocal tumors or tumors affecting the neck of the pancreas, a complete pancreatectomy can also be necessary.

\section{Pancreaticoduodenectomy}

The classic Whipple pancreaticoduodenectomy includes resection of the head of the pancreas, uncinate process, and right-sided body of the pancreas as well as of the duodenum, bile duct, proximal jejunum, and the stomach pylorus and antrum. A variant of this procedure is pylorus-preserving pancreaticoduodenectomy using the Traverso-Longmire technique which differs from the Whipple procedure with respect to the preservation of the distal stomach. As a result of the removal of the above-mentioned structures, an anastomosis between the remaining left-sided pancreas and the proximal jejunum (pancreaticojejunostomy) and between the centrally dissected bile duct in the hepatic hilum and the jejunum (hepatocholedochojejunostomy) is created. Diges- tion is ensured either via gastrojejunostomy (classic Whipple procedure) with optional placement of a Braun's enteroanastomosis or via duodenojejunostomy (using the Traverso-Longmire technique). In the case of local tumor infiltration, portal venous vascular reconstruction, which is routinely offered by most centers, is sometimes necessary. Arterial resection is reserved for special cases. Moreover, locoregional lymphadenectomy is performed.

\section{Left-sided pancreatic resection}

In the case of pancreatic tumors in the left-sided distal body of the pancreas or tail of the pancreas, left-sided pancreatic resection/ resection of the tail of the pancreas can be performed. The left-sided pancreas is dissected in the region of the body of the pancreas with a safety distance to avoid the need for complicated anastomoses. As a rule, en-bloc removal of the spleen and a corresponding lymphadenectomy are performed under oncological conditions. 
- Table 4 Absolute and relative surgical indications for intraductal papillary mucinous neoplasm (IPMN) [6].

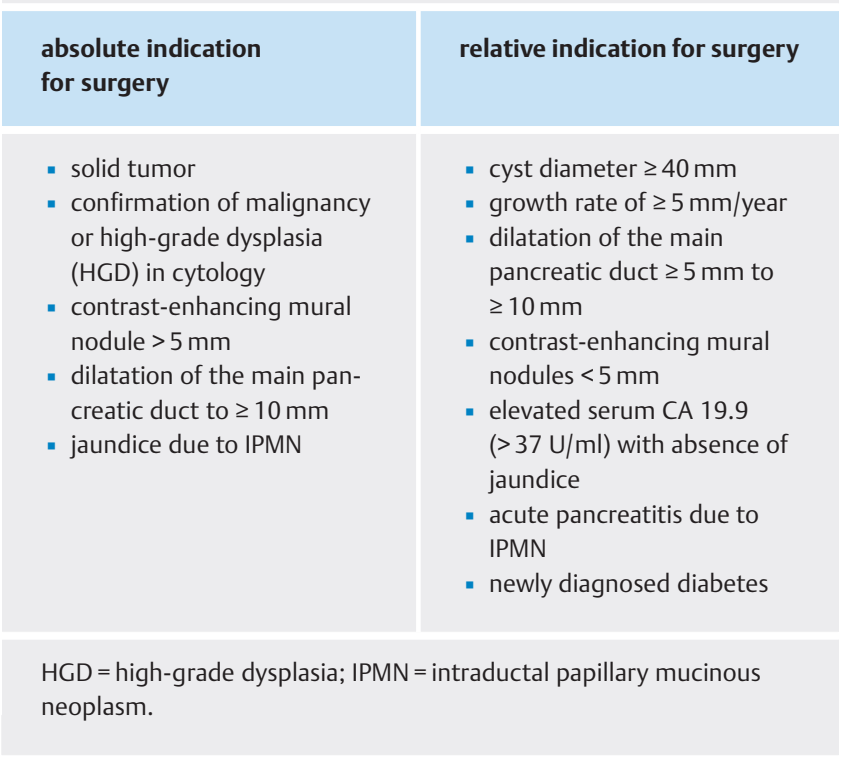

\section{Summary}

The structured report templates presented here for solid and cystic pancreatic tumors are intended to improve the quality of radiology reports and to optimize interdisciplinary communication in the radiological routine.

\section{Conflict of Interest}

The authors declare that they have no conflict of interest.

\section{References}

[1] Pinto Dos Santos D, Hempel JM, Mildenberger P et al. Structured Reporting in Clinical Routine. Rofo 2019; 191: 33-39. doi:10.1055/a-0636-3851

[2] Schwartz LH, Panicek DM, Berk AR et al. Improving communication of diagnostic radiology findings through structured reporting. Radiology 2011; 260: 174-181. doi:10.1148/radiol.11101913
[3] Brook OR, Brook A, Vollmer CM et al. Structured reporting of multiphasic CT for pancreatic cancer: potential effect on staging and surgical planning. Radiology 2015; 274: 464-472. doi:10.1148/radiol.14140206

[4] Kabadi S], Krishnaraj A. Strategies for Improving the Value of the Radiology Report: A Retrospective Analysis of Errors in Formally Over-read Studies. J Am Coll Radiol 2016. doi:10.1016/j.jacr.2016.08.033

[5] Al-Hawary MM, Francis IR, Chari ST et al. Pancreatic ductal adenocarcinoma radiology reporting template: consensus statement of the Society of Abdominal Radiology and the American Pancreatic Association. Radiology 2014; 270: 248-260. doi:10.1148/radiol.13131184

[6] Pancreas ESGoCTot. European evidence-based guidelines on pancreatic cystic neoplasms. Gut 2018; 67: 789-804. doi:10.1136/gutjnl-2018316027

[7] Qayyum A, Tamm EP. Expert Panel on Gastrointestinal I,. et al. ACR Appropriateness Criteria((R)) Staging of Pancreatic Ductal Adenocarcinoma. J Am Coll Radiol 2017; 14: S560-S569. doi:10.1016| j.jacr.2017.08.050

[8] Tempero MA, Malafa MP, Al-Hawary M et al. Pancreatic Adenocarcinoma, Version 2.2017, NCCN Clinical Practice Guidelines in Oncology. | Natl Compr Canc Netw 2017; 15: 1028-1061. doi:10.6004/ jnccn.2017.0131

[9] Ducreux M, Cuhna AS, Caramella C et al. Cancer of the pancreas: ESMO Clinical Practice Guidelines for diagnosis, treatment and follow-up. Ann Oncol 2015; 26 (Suppl. 5): v56-v68. doi:10.1093/annonc/mdv295

[10] Seufferlein T, Porzner M, Becker T et al. S3-guideline exocrine pancreatic cancer. Z Gastroenterol 2013; 51: 1395-1440. doi:10.1055/s-00331356220

[11] Tempero MA, Malafa MP, Chiorean EG et al. Pancreatic Adenocarcinoma, Version 1.2019. J Natl Compr Canc Netw 2019; 17: 202-210. doi:10.6004/jnccn.2019.0014

[12] Buerke B, Heindel W, Wessling J. Differential diagnosis and radiological management of cystic pancreatic lesions. Rofo 2010; 182: 852-860. doi:10.1055/s-0029-1245502

[13] Brambs HJ, Juchems M. Cystic tumors of the pancreas. Radiologe 2008; 48: 740-751. doi:10.1007/s00117-008-1671-4

[14] Schlitter AMEE, Klöppel G. Klassifikation und Diagnose zystischer Pankreastumoren. Gastrointestinal Medicine and Surgery 2011; 27: 182 188. doi:10.1159/000329194

[15] Buerke B, Domagk D, Heindel W et al. Diagnostic and radiological management of cystic pancreatic lesions: important features for radiologists. Clin Radiol 2012; 67: 727-737. doi:10.1016/j.crad.2012.02.008

[16] Arnold et al. Pankreas. In: Frenzel, Hrsg. Kap. 3.5. Tumorerfassung. Thieme; 2012; 1. Aufl

[17] Grenacher L, Juchems M. Criteria for resectability of pancreatic cancer and postoperative imaging. Radiologe 2017; 57: 1075-1090. doi:10.1007/s00117-017-0326-8 